\title{
Da Milano a San Paolo
}

Rafael Sozzi (mestrando USP) rafaelsozzi@yahoo.com.br

Abstract: Destino, destinazione, partenza, viaggio sono parole che trovano un denominatore comune nel fenomeno della migrazione. Soprattutto, per quanto riguarda la migrazione verso il Nuovo Mondo. Il Nuovo Mondo è vita nuova. È avere un'altra possibilità di essere protagonista del proprio destino. L'immigrazione italiana in America Latina è caratterizzata, soprattutto, per la creazione e costruzione di una nuova identità e per la collaborazione alla costituzione di nuove nazioni.

Key words: Viaggio. Immigrazione. Italia. America Latina.

Resumo: Destino, destinação, partida, viagem são palavras que se encontram no fenômeno da imigração. Especialmente no que diz respeito ao Novo Mundo que representa uma Vida Nova e a possibilidade de ser protagonista do próprio destino. A imigração italiana para a América latina é caracterizada, de modo particular, pela criação e construção de uma nova identidade e pela colaboração dada à construção de novas nações.

Palavras-chave: Viagem. Imigração. Itália. América Latina.

In letteratura è significativo il numero di protagonisti che realizzano un viaggio. Basti pensare all'Odissea, all'Eneide, ai Lusíadas. Oppure al viaggio attraverso l'Inferno, il Purgatorio e il Paradiso; o più vicini a noi il Grand Tour e i viaggi nelle città e nelle campagne europee. Ma esiste un altro viaggio senza ritorno.

Destino, destinazione, partenza, viaggio. Parole che trovano un denominatore comune nel fenomeno della migrazione. Soprattutto, per quanto riguarda la migrazione verso il Nuovo Mondo. Un mondo in tutto e del tutto nuovo, da costruire, da creare. Un mondo nuovo che nella mente di un giovane ventisettenne (la cui lettera leggiamo qui) è in puro contrasto con il Vecchio Mondo, in cui guerre, dittatura, morte, sofferenze, privazioni risuonano ancora come un tormento infinito, indimenticabile. Il Nuovo Mondo è vita nuova. È 
ricostruirsi, è rinascere, è avere un'altra possibilità di compiere il proprio destino. Anzi, di essere protagonista del proprio destino.

Studiosi dellimmigrazione italiana in America Latina sottolineano che questo fenomeno, diversamente da altri casi di migrazione italiana, è caratterizzato, soprattutto, per la creazione e costruzione di una nuova identità, per la collaborazione alla costituzione di nuove nazioni. In un paese come il Brasile, multietnico e multiculturale, gli italiani hanno contribuito, insieme ad altri popoli, a costruire una nazione, una cultura, un'identità. Non per caso è una delle "etnie" più presenti nel territorio brasiliano (si presume che circa 6 milioni sono gli italiani e oriundi residenti nella città di San Paolo e 13 milioni in tutto lo Stato di San Paolo).

Nella prima lettera (Fig. 1) che proponiamo è possibile riscontrare un bellissimo esempio di ciò che abbiamo detto sopra. Indirizzata a Renzo Sozzi, nel 1948, da parte di suo zio Augusto Pisati, immigrato in Brasile nel 1923, questa lettera ci rivela l'aspetto non stereotipato dell'emigrazione. Non tutti partono con la valigia di cartone per scampare dai mali, dai problemi di un paese senza prospettive, luoghi comuni ormai costanti nelle ricerche sull'emigrazione italiana. È invece l'emigrazione verso il nuovo, ma non soltanto per motivazioni puramente economiche, redditizie, bensì per la possibilità di "concretizzare le proprie aspirazioni”; di "risolvere quei problemi (problemi d'amore, perché Renzo si era innamorato di una ragazza di origine italiana che viveva a San Paolo) già programmati nel "taquino (taccuino) della vita". E quindi, l'occasione di farsi una famiglia, di costruire un patrimonio. Proprio come il Renzo Tramaglino dei Promessi Sposi, che - dopo tutte le sorti subite per compiere il suo destino, cioè ritrovare e sposare Lucia - lascia il solo natio perché è questa l'unica possibilità di stare con la persona amata. Nello scritto dunque viene confermato l'acquisto del biglietto per il viaggio, l'attesa dei parenti ansiosi di conoscere il cugino "italiano", l'incoraggiamento della decisione di migrare.

Lo scritto di Renzo (Fig. 2) invece mostra tutte le difficoltà ed i dubbi di un'eventuale partenza. Nel maggio 1948, prima ancora di essere consapevole 
dell'eminente viaggio, Renzo scrive a Renata perché ormai era già chiaro per entrambi il vicendevole sentimento di stima. L'amicizia nata nel viaggio di lei in Italia, nel 1947, va oltre l'oceano che li separa. I pochi mesi di conoscenza - segnati però da incontri, balli, pranzi, cene, gite, litigi ecc. - sono intensi e quindi condizionano il proseguimento della loro vita. È difficile partire. Ma è difficile anche l'assenza di lei.

\footnotetext{
Mi è caro ricordare le ore trascorse insieme a te ed agli zii, ore belle, allegre, sane; ore in cui lo spirito si apriva alla gioia più limpida dell'affetto e della simpatia ed è veramente doloroso che la vita costringa a rinunciare a queste gioie, ponendo gli affetti a distanza di centinaia di chilometri! Anche se si [rinasce] nel cuore ogni ricordo e l'affetto tutto, la gioia della compagnia fisica e della conversazione e molto è il dolore che ne deriva. Io già penso anche a ciò che proverò lontano dai miei cari nell'eventualità della mia partenza. Sarà doloroso vivere lontano dalle persone più care strozzando nello spirito il continuo desiderio d'averli vicini e dimenticando a viva forza i giorni belli del loro affetto.
}

Decidere poi non è l'unica difficoltà. Sarà vero questo amore? Si tratta di un amore reciproco. Non si può abbandonare tutto. Rischiare senza avere nemmeno la certezza se l'amore è corrisposto.

E tu Renata perché non parli con me più intimamente come si suol fare con un amico del quale si conosce la sicura devozione? La tua vita non è e non sarà fatta soltanto della scuola, dei balli e delle compagne, e dunque? Nessuna cosa desideri da questa Italia che tanto t'è piaciuta? Nessun ricordo particolarmente ritorna alla tua mente senza che tu senta di [rinvocarlo] con qualcuno?

Renzo dunque decide di partire. Lascia Milano con destinazione San Paolo non solo per motivi lavorativi (lavorerà come impiegato presso la Metalúrgica Mercúrio, azienda fondata dagli zii Augusto e Arturo Pisati negli anni '30), ma soprattutto per ritrovare colei che gli aveva creato delle inquietudini; e per essere, forse per la prima volta, il protagonista della propria vita, del proprio destino. 
Queste lettere fanno parte di un corpus di scritti (circa 200 lettere manoscritte e dattiloscritte) che testimoniano sia le molteplici caratteristiche dell'immigrazione italiana in Brasile sia l'uso della lingua italiana in territorio brasiliano. Lo studio dell'epistolografia e di altri tipi di registri si inserisce in un lavoro più ampio di ricerca e di mappatura dell'uso dell'italiano in Brasile, promosso dall'Universidade de São Paulo (USP). Perciò uno degli scopi principali della nostra ricerca è quello di analizzare, e quindi descrivere, i fenomeni linguistici che caratterizzano la lingua italiana parlata e scritta da una piccola comunità lombarda, residente a San Paolo dall'inizio del XX secolo. La lingua italiana e le comunità che ne facevano uso nella capitale paulista, contribuirono alla formazione linguistica e culturale della città. Studiare l'italiano e le sue varietà al di fuori dei confini italiani, e dunque in Brasile, è necessario per comprendere meglio il suo ruolo nella formazione del portoghese brasiliano.

Siamo conviti che lo studio della lingua italiana utilizzata dagli immigrati può chiarire non solo le modalità dell’immigrazione - poiché in questi registri sono riscontrabili testimonianze dettagliate dei viaggi, dei sentimenti, delle aspettative ecc. - ma anche quali erano le caratteristiche di queste persone che, mossi da una forte speranza di un futuro migliore, lasciavano le loro case per avventurarsi nel Nuovo Mondo, molte volte consapevoli dei rischi e dei luoghi comuni che permeavano la nuova vita in Brasile. 
Hosulo 22 agoots 918 Cansicino Benco

quando riceverai questa meia mistica, fow cesto the tutte le campone di Wilono Jusneranno a storno. dicendor finahnente a veritto Kio Anqusto now è Vaso?

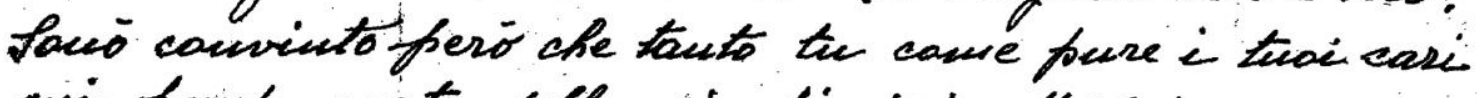
mi perdonerete della mia pigrisia di desivese. Delle voptre rotirie, is era becifre al corrente dalle

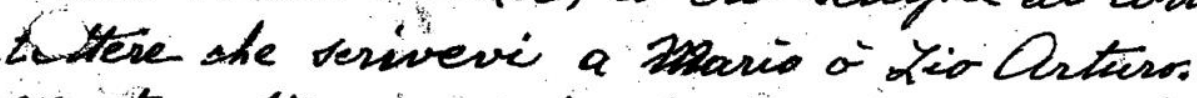
Mentre to sivivendo, sento che oggi arrivera a Gemova if vapore Brasile, insuaginiamo la sootra

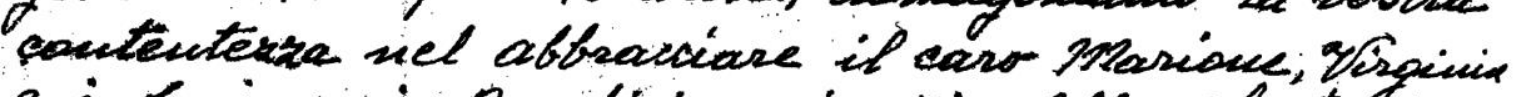

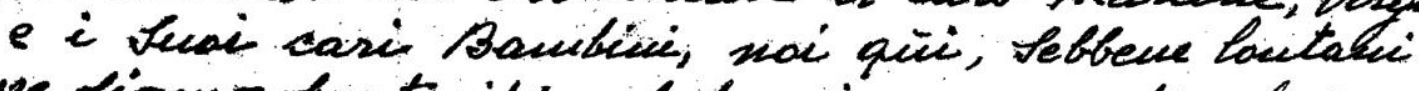
ne diano partecipi col pew iers a questo fansto ovemiments, - Speriano che abbius fatto in busic viaggios

Gioreci on buo to sicevecto un tio soritto, insience ad una lettera invata alb Yio Orturo. Q. ringraitio delle belbiriune parale di affett versa

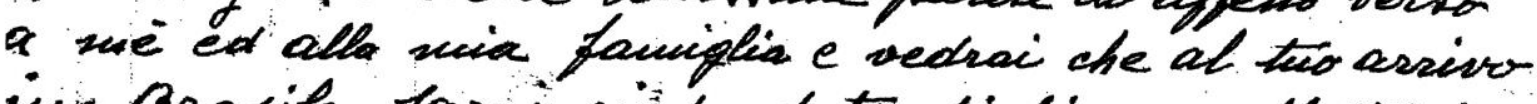
in Brasite toirat sindoudato di tiucero affeth da. titt. i miei cari, she vebbue man ti canosenus Hentama gia la giaia di abraciarti presto. in gabiua de Its clave nella wave datia, cosi 
vressei insience a Mario, vei costento? il bigliett col

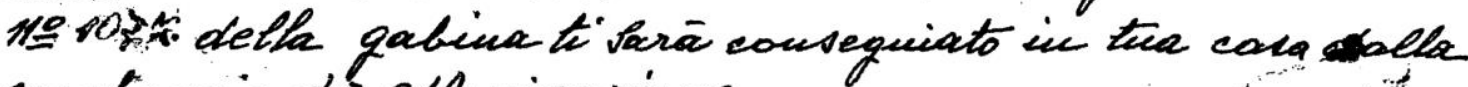
sarofiaquia de elaviganiaue.

Sd" ora caro Benzo ti aspettionu a brassia Opesits. ba decizione di venire in Brasile fer insoninciase una vite fier sendibiria e per sisolvere quei froblemin gia frograne nati nel taquino della tua vita e che stamo fris al tur cuare, tho la certerza che eolla tiva inteligeuse e buona - loutai di lavero patrociucato della usotra esferieura $e$ del nosto aiuto siuscirai a coucretirpare be the aspirasiani.

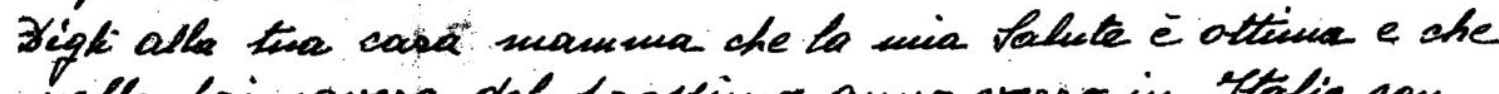
nella prinuavere det hrossino anno verso in Htalia can la una faninglin, meno bttare, che uon fuo hasciase i Husi studi) sosi dopo 25 ouni di Brasizile mi Lasa

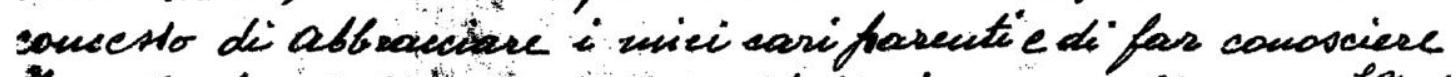

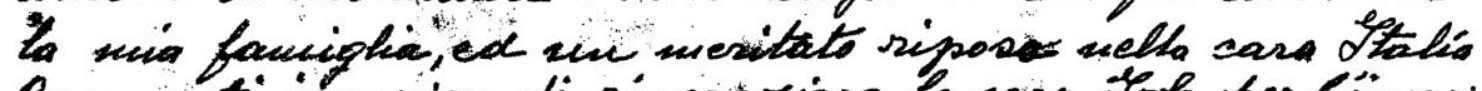
Bruna ti insarisa di riugrasiare la sarn Lote per lüusariso

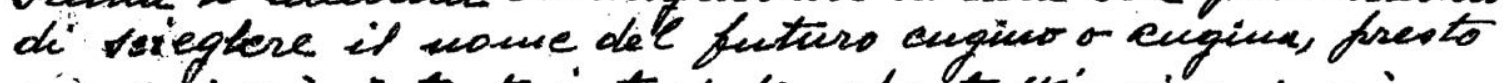
9." Jorivera, è tuto intusiata, she tutte" giosui sui dowanda! Tarai vero papa she audiano in Stalis?

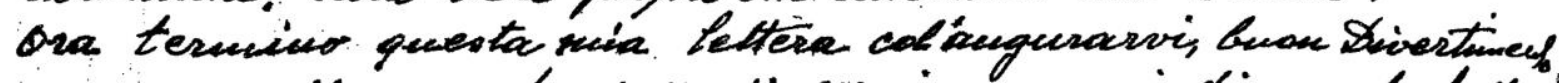

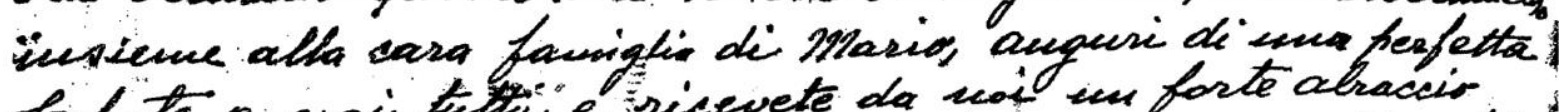

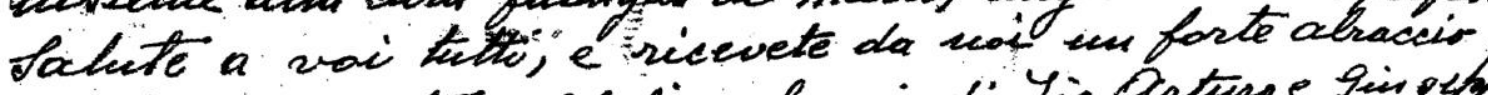

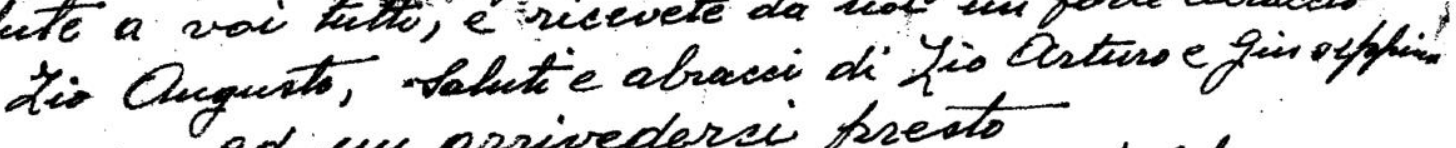
ed un arrivedersi presto

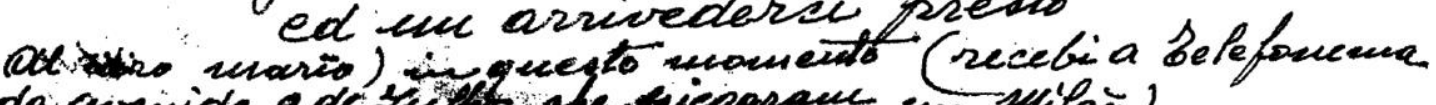

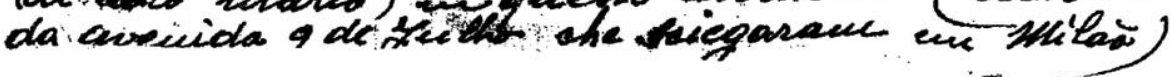

Fig. 1 - lettera di Augusto Pisati al nipote Renzo Sozzi 


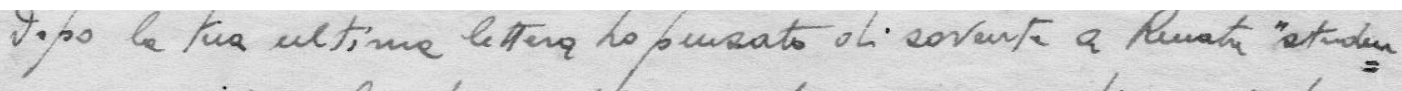

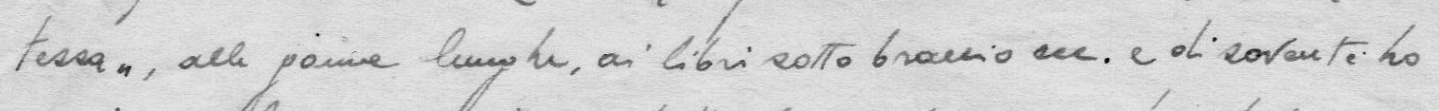

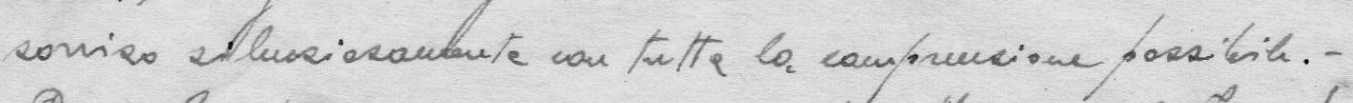

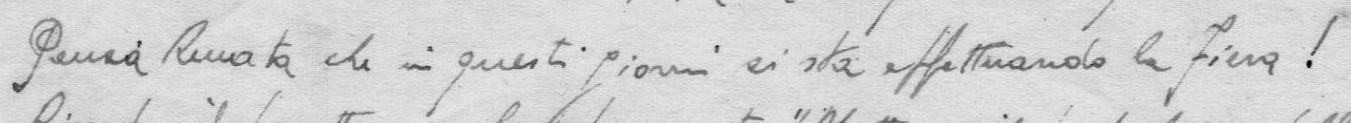

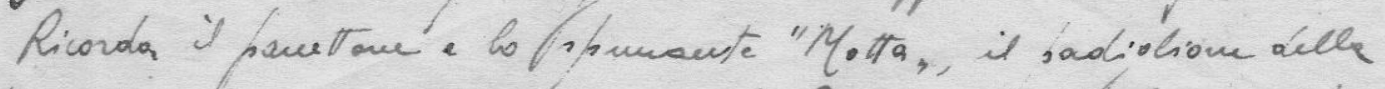

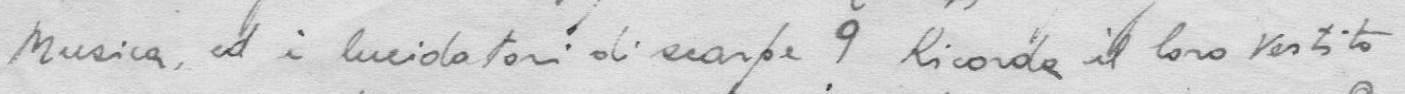

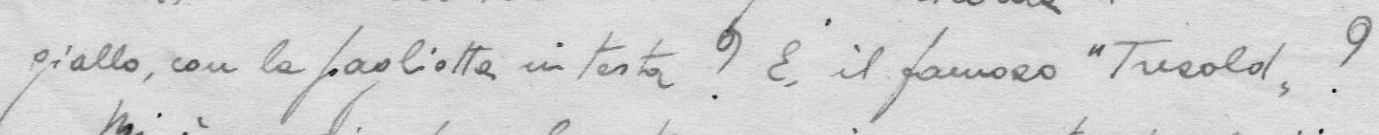
In' icaro hicordan fe ou traxeonse insieme a te ed agkinis; on

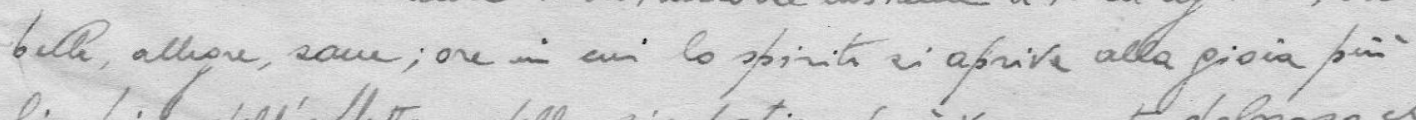

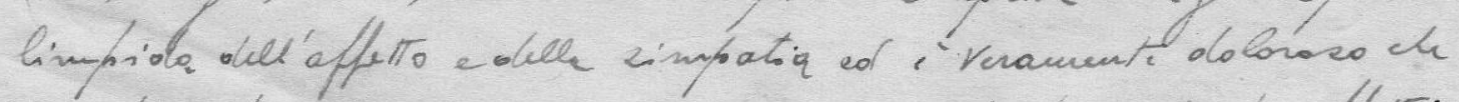

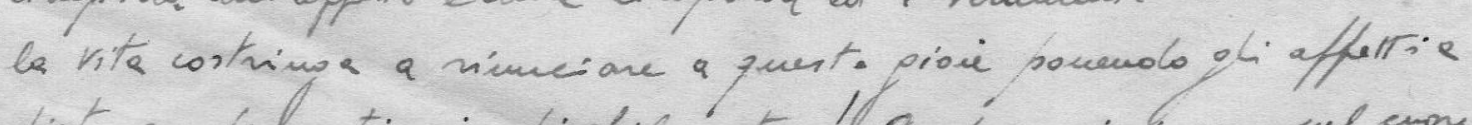

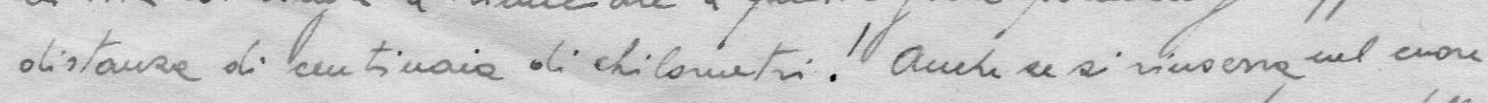

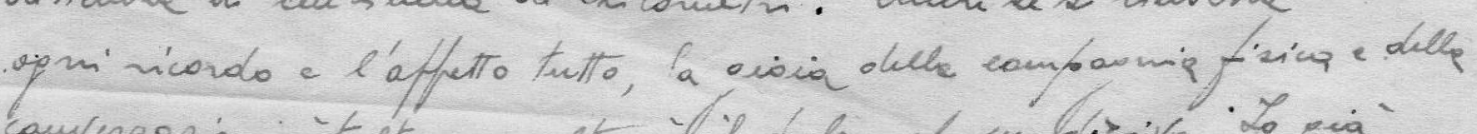

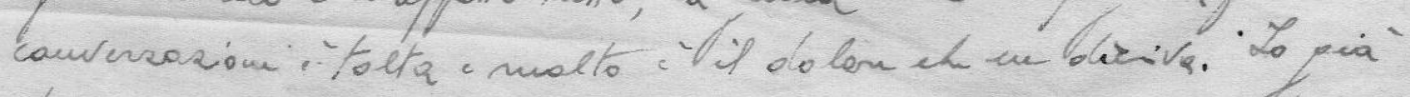

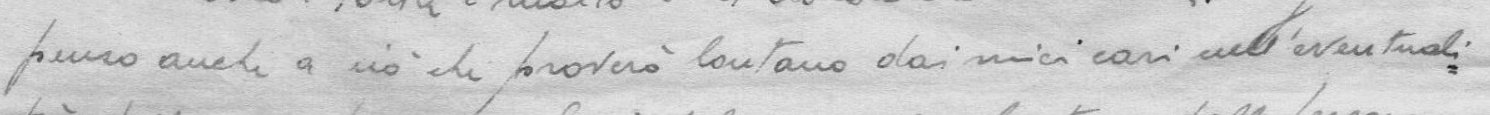

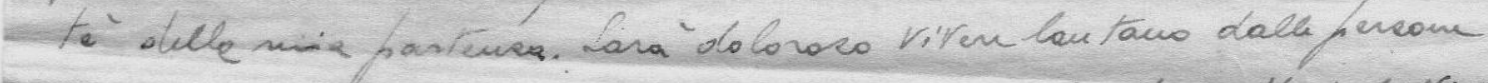

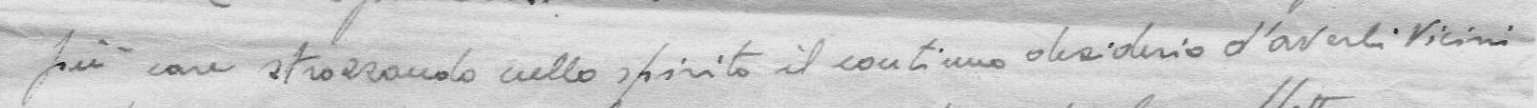

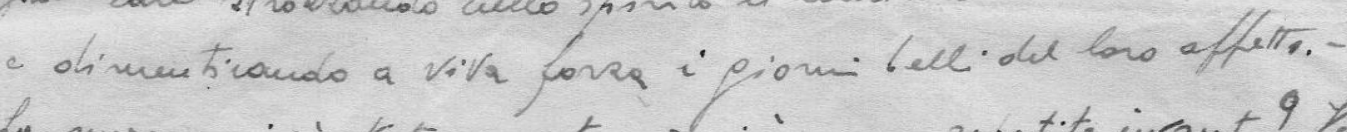

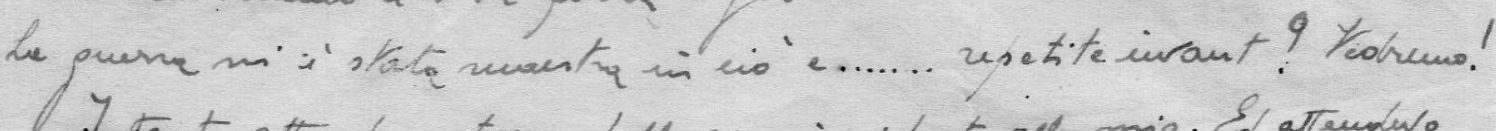

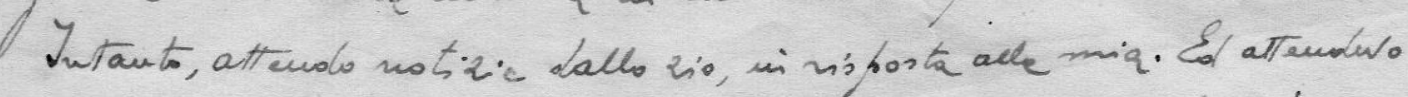

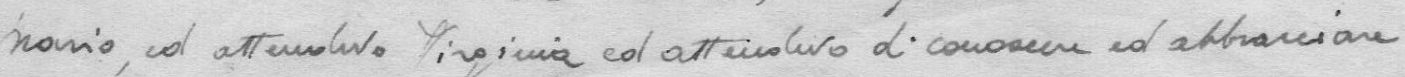

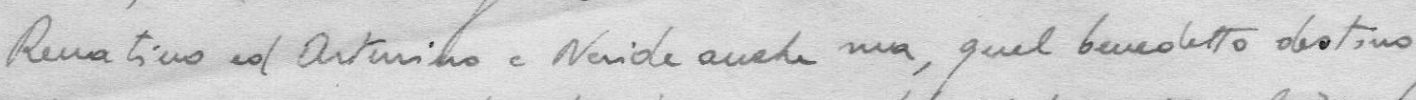

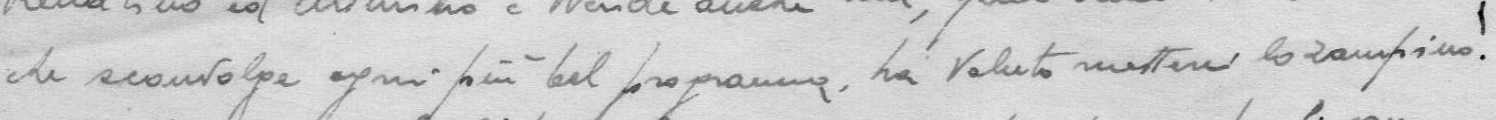

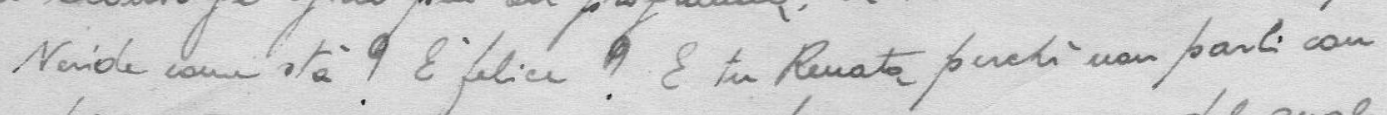

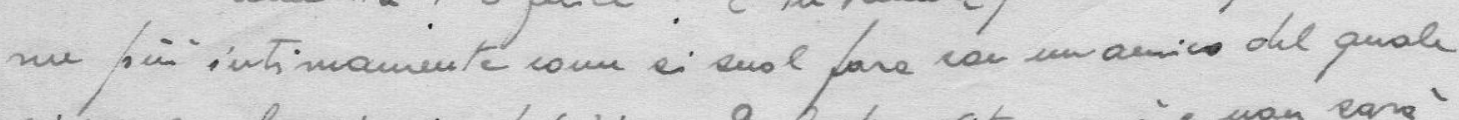

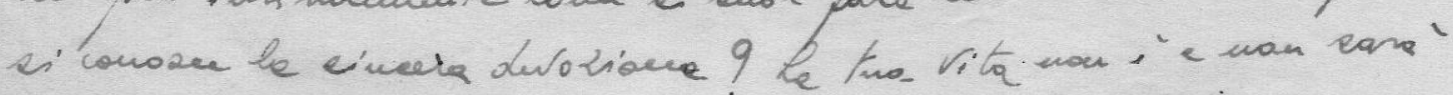

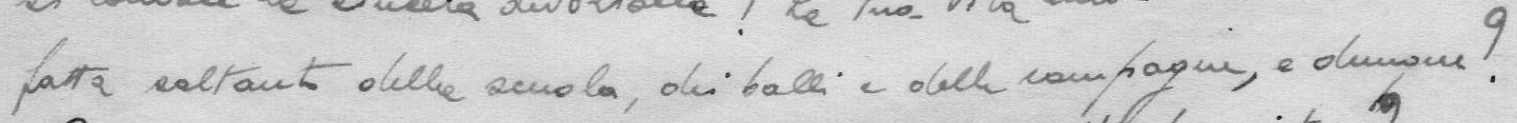

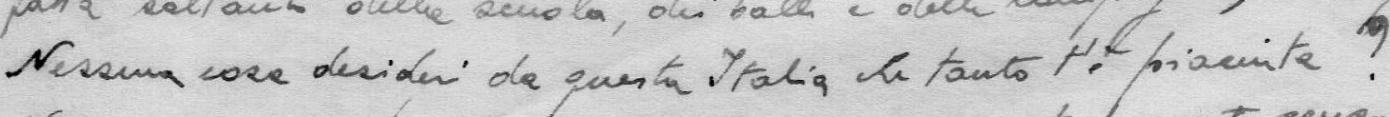

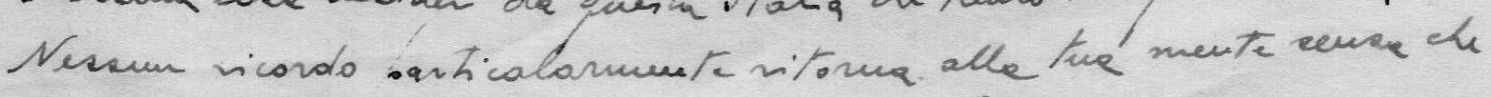
tur enta oli tivecarlo can sualemus?

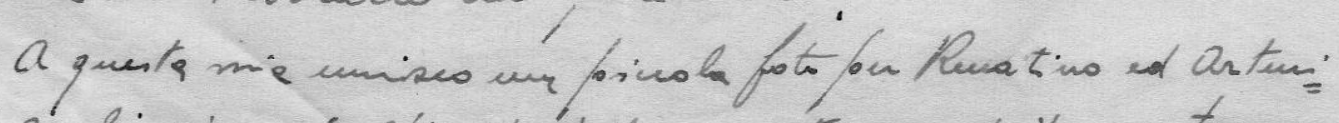

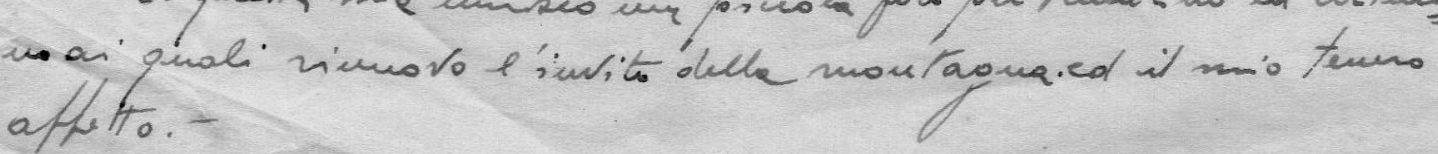

